\title{
Location Description
}

National Cancer Institute

\section{Source}

National Cancer Institute. Location Description. NCI Thesaurus. Code C93589.

The textual representation of the physical location of the subject or entity. 